Copyright ( 2013 IEEE. Personal use of this material is permitted. Permission from IEEE must be obtained for all other uses, in any current or future media, including reprinting/republishing this material for advertising or promotional purposes, creating new collective works, for resale or redistribution to servers or lists, or reuse of any copyrighted component of this work in other works. 


\section{Intrinsic Cross-Polarization Ratio of Dual-Linearly Polarized Antennas for Low-Frequency Radio Astronomy}

\author{
Adrian T. Sutinjo and Peter J. Hall
}

\begin{abstract}
This note discusses the Intrinsic Cross-Polarization Ratio (IXR) from an antenna engineering perspective in that we seek to identify an a priori (coordinate) system where IXR is well approximated by the raw cross-polarization numbers. We begin by establishing a special case where IXR is identical to the raw cross-polarization ratios for in-phase dual-linearly-polarized antennas when the Jones matrix is expressed using circular polarization bases. This insight allows physical interpretation of IXR which may be useful in antenna design and system calculations. In addition, we discuss comparisons between direct IXR calculations and circular polarization approximations for more realistic cases involving dual-polarized Murchison Widefield Array (MWA) bow-tie antennas.
\end{abstract}

Index Terms- Antenna theory, Radio astronomy, Polarimetry

\section{INTRODUCTION}

The study of polarization purity of dual-polarized antennas for radio astronomy polarimetry involves detailed knowledge on two primary fronts: antenna design and polarimetric calibration [1]-[3]. While the exact boundary demarcating these areas is not easily discernible, it is generally agreed that polarimetric calibration removes raw antenna polarization impurity to a large degree [4], [5]. This perception, however, leaves the issue of polarization purity of the dual-polarized antenna rather vague-how this parameter should be specified seems unclear.

A recent development in radio polarimetry suggests a fundamental figure of merit (FoM) - the intrinsic cross-polarization ratio-which is commensurate with the condition number of the Jones matrix of the polarimeter [4], [5]. The Jones IXR ${ }^{1}$ is defined as

$$
\mathrm{IXR}=\left(\frac{\sigma_{\max } / \sigma_{\min }+1}{\sigma_{\max } / \sigma_{\min }-1}\right)^{2}
$$

where $\sigma_{\max }$ and $\sigma_{\min }$ refer to the maximum and minimum singular values of the Jones matrix and $\sigma_{\max } / \sigma_{\min }=$ $\|\mathbf{J}\|_{2}\left\|\mathbf{J}^{-1}\right\|_{2}$ is the (spectral norm) condition number for the Jones matrix [5]. The IXR appears advantageous as it provides a FoM that is independent of coordinate systems ${ }^{2}$ and provides an upper bound estimate for total relative error of the sky Jones vector $^{3}$ after calibration [5], [7].

$$
\frac{\|\Delta \mathbf{e}\|}{\|\mathbf{e}\|} \lesssim\left(1+\frac{2}{\sqrt{\mathrm{IXR}}}+\ldots\right)\left(\frac{\|\Delta \mathbf{J}\|}{\|\mathbf{J}\|}+\frac{\|\Delta \mathbf{f}\|}{\|\mathbf{f}\|}\right)
$$

Rev. 1 Final, January 21, 2013.

The authors are with Curtin Institute of Radio Astronomy, Curtin University, Bentley, WA 6102, Australia (e-mail: adrian.sutinjo@gmail.com).

${ }^{1}$ We limit our discussions here to the Jones IXR.

${ }^{2}$ As matrix spectral norm is unitarily invariant [6] and coordinate transformations matrices are unitary.

${ }^{3}$ The term "sky Jones vector" or "sky vector" refers to the polarization state of the incoming electric field from a particular direction in the sky. "Measured/measurement vector" refers to the voltages or currents measured at the ports of the dual-polarized antenna. where $\|\Delta \mathbf{J}\| /\|\mathbf{J}\|$ and $\|\Delta \mathbf{f}\| /\|\mathbf{f}\|$ are the relative errors for the calibrated Jones matrix and the measured vector, respectively. Note that IXR $=26 \mathrm{~dB}$ and $20 \mathrm{~dB}$ equate to $2 / \sqrt{\mathrm{IXR}}=0.2$ and 0.1 , respectively (indicating upper bounds for total relative error magnification of $20 \%$ and $10 \%)^{4}$.

In principle, assuming an experiment for which the required IXR is known, the antenna polarization purity may be specified in terms of IXR [4], [5]. However, although the IXR is easily computed after the fact for a given antenna, the process of antenna synthesis typically requires an a priori intuition to contrive a promising design starting point. The latter seems lacking at the moment as physical interpretation for IXR is not immediately apparent. Consequently, the motivation for this communication is to find a familiar frame of reference in antenna engineering where the IXR is obtained exactly or is well approximated ${ }^{5}$. In particular, we examine the case of dipole-like antennas (specifically, the MWA bow-tie [9], [10]) over a ground plane which have been adopted in many lowfrequency radio astronomy arrays [11]-[14]. Topics involving derivation of IXR requirements for a particular scientific experiment and polarimetric calibration are pertinent ones, however, are beyond our current scope. Our focus here is in providing a means for physically interpreting IXR as it relates to dual-polarized linear antennas.

This communication is organized as follows. Section II discusses the IXR and polarization purity of dual-linearlypolarized antennas. Calculated results and conclusion are presented in Sections III and IV, respectively.

\section{IXR OF DUAL-LINEARLY-POLARIZED ANTENNAS}

\section{A. Circular polarization bases}

In defining polarization purity for antennas of arbitrary orientation, circular polarization $(\mathrm{CP})$ is preferred over linear polarization (LP) as antenna misorientation results in phase but not amplitude change [15]. This is applicable to low-frequency radio astronomy as the source positions are arbitrary relative to fixed antennas. In the following, we point out the conditions that lead to a special case where the raw cross-polarization (XP) ratios are identical to IXR for dual-linearly-polarized antennas.

The relationship between the measured vector, Jones matrix, and sky vector of a dual-linearly polarized antenna system at a particular frequency and direction $(\theta, \phi$ in the spherical coordinate system) is given by

$$
\mathbf{f}_{x^{\prime} y^{\prime}}=\mathbf{J}_{L} \mathbf{e}_{\theta \phi}
$$

where $\mathbf{f}_{x^{\prime} y^{\prime}}=\left(f_{x^{\prime}}, f_{y^{\prime}}\right)^{T}$ indicates the measured vector, $\mathbf{e}_{\theta \phi}=$

\footnotetext{
${ }^{4}$ Some readers may be more familiar with the condition number which is also a measure of the upper bound for the relative error in the output divided by the relative error in the input [8]. The condition number needed to solve a polarimetry problem $\mathbf{e}=\mathbf{J}^{-1} \mathbf{f}$ depends on the accuracy with which $\mathbf{J}$ and $\mathbf{f}$ may be determined via measurement and calibration (note- as pointed out in [3] - that calibration processes using an unpolarized source depend on inherent polarization quality of the antenna system). For instance if this accuracy is $10^{-1}$, then the condition number needs to be much less than 10 .

${ }^{5} \mathrm{An}$ antenna designer faced with having to meet $\mathrm{x}$ dB IXR will likely find these "clues" helpful
} 
$\left(e_{\theta}, e_{\phi}\right)^{T}$ denotes the sky Jones vector, and

$$
\mathbf{J}_{L}=\left(\begin{array}{cc}
J_{x^{\prime} \theta} & J_{x^{\prime} \phi} \\
J_{y^{\prime} \theta} & J_{y^{\prime} \phi}
\end{array}\right)
$$

is the Jones matrix. Note that the superscript ' in $x^{\prime}$ and $y^{\prime}$ indicates that the orientation of the linearly polarized antennas are only nominal (i.e., both misalignment from true $\mathrm{x}$ and $\mathrm{y}$ as well as non-orthogonal antennas are allowed).

Changing the measurement basis to nominally CP and sky basis to $\mathrm{CP}$ results in

$$
\mathbf{J}_{C}=\frac{1}{2}\left(\begin{array}{cc}
J_{l^{\prime} l} & J_{l^{\prime} r} \\
J_{r^{\prime} l} & J_{r^{\prime} r}
\end{array}\right)=\mathbf{R}_{C} \mathbf{J}_{L} \mathbf{R}_{C}^{H}
$$

where again the the superscript ${ }^{\prime}$ in $l^{\prime}$ and $r^{\prime}$ indicates nominal left-hand $\mathrm{CP}$ and right-hand $\mathrm{CP}$, respectively, and

$$
\mathbf{R}_{C}=\frac{1}{\sqrt{2}}\left(\begin{array}{cc}
-j & 1 \\
1 & -j
\end{array}\right)
$$

is a transformation matrix (unitary) from LP to CP bases and the superscript ${ }^{H}$ indicates conjugate transpose.

Expressing the elements of $\mathbf{J}_{C}$ in terms of the elements of $\mathbf{J}_{L}$ we obtain

$$
\begin{aligned}
& J_{l^{\prime} l}=J_{x^{\prime} \theta}+J_{y^{\prime} \phi}+j\left(J_{y^{\prime} \theta}-J_{x^{\prime} \phi}\right) \\
& J_{l^{\prime} r}=J_{x^{\prime} \phi}+J_{y^{\prime} \theta}+j\left(J_{y^{\prime} \phi}-J_{x^{\prime} \theta}\right) \\
& J_{r^{\prime} l}=J_{x^{\prime} \phi}+J_{y^{\prime} \theta}+j\left(J_{x^{\prime} \theta}-J_{y^{\prime} \phi}\right) \\
& J_{r^{\prime} r}=J_{x^{\prime} \theta}+J_{y^{\prime} \phi}+j\left(J_{x^{\prime} \phi}-J_{y^{\prime} \theta}\right)
\end{aligned}
$$

In (7), if $\mathbf{J}_{L}$ is a real matrix, i.e., the dual-polarized antennas are entirely linearly polarized (axial ratio $=\infty$ ) with no phase offset between the measurements, $\mathbf{J}_{C}$ takes the form

$$
\mathbf{J}_{C}^{\prime}=\frac{1}{2}\left(\begin{array}{cc}
J_{l^{\prime} l} & J_{l^{\prime} r} \\
J_{l^{\prime} r}^{*} & J_{l^{\prime} l}^{*}
\end{array}\right) \quad \text { for } \operatorname{Im}\left(\mathbf{J}_{L}\right)=0
$$

In (8) note that the raw cross-polarization isolations (XPI) and discriminations (XPD) are equal to one another.

$$
\begin{aligned}
& \mathrm{XPI}_{l^{\prime}}=\left|\frac{J_{l^{\prime} l}}{J_{l^{\prime} r}}\right|^{2}=\mathrm{XPI}_{r^{\prime}}=\left|\frac{J_{l^{\prime} l}^{*}}{J_{l^{\prime} r}^{*}}\right|^{2}= \\
& \mathrm{XPD}_{l}=\left|\frac{J_{l^{\prime} l}}{J_{l^{\prime} r}^{*}}\right|^{2}=\mathrm{XPD}_{r}=\left|\frac{J_{l^{\prime} l}^{*}}{J_{l^{\prime} r}}\right|^{2}=\mathrm{XP}
\end{aligned}
$$

Furthermore, it can be shown that the singular values of $\mathbf{J}_{C}^{\prime}$

$$
\begin{aligned}
\sigma & =\sqrt{\lambda\left(\mathbf{J}_{C}^{\prime H} \mathbf{J}_{C}^{\prime}\right)} \\
& =\frac{1}{2}\left|J_{l^{\prime} l}\right|\left(1 \pm \frac{1}{\sqrt{\mathrm{XP}}}\right)
\end{aligned}
$$

where $\lambda()$ indicates the eigenvalues of the matrix in question. Using (1) we find that for the special case $\mathbf{J}_{C}=\mathbf{J}_{C}^{\prime}$ shown in $(8)$

$$
\mathrm{IXR}=\mathrm{XP}
$$

Thus, we have achieved the stated objective, that is to define the conditions for dual-linearly-polarized antennas such that raw cross-polarizations are identical to IXR. It should be pointed out that the condition $\operatorname{Im}\left(\mathbf{J}_{L}\right)=0$ is met for many textbook antennas such as dipoles and loops [16]. Thus (11) applies to system level calculations involving such antennas. Note that although we started the derivation with linearly polarized antennas, this finding is equally applicable to any dual-polarized antenna whose Jones matrix takes the form shown in (8).

\section{B. Factors that affect IXR approximation}

The assumption $\operatorname{Im}\left(\mathbf{J}_{L}\right)=0$ is pivotal in obtaining (11). Accordingly, as the imaginary parts of the elements in $\mathbf{J}_{L}$ become non-negligible, IXR approximation using $\mathrm{CP}$ bases becomes less accurate. We comment on this briefly here, followed by calculated examples in the next section.

1) Consider phase imbalance for $x^{\prime}$ measurement represented by

$$
\mathbf{P}_{x^{\prime}}=\left(\begin{array}{cc}
e^{j \varphi} & 0 \\
0 & 1
\end{array}\right)
$$

The total Jones matrix is a complex matrix given by

$$
\mathbf{J}=\mathbf{P}_{x^{\prime}} \mathbf{J}_{L}^{r e a l}=\left(\begin{array}{cc}
J_{x^{\prime} \theta} e^{j \varphi} & J_{x^{\prime} \phi} e^{j \varphi} \\
J_{y^{\prime} \theta} & J_{y^{\prime} \phi}
\end{array}\right)
$$

Note that as (12) is a unitary matrix, IXR remains unchanged. However, $\mathbf{R}_{C} \mathbf{J R}_{C}^{H}$ no longer has the form shown in (8) such that IXR $\neq$ XP. This situation occurs in practice as dual-polarized antennas will not be exactly identical. In this case, $\mathrm{CP}$ approximation will be markedly improved by simple phase compensation (more on this in the next section).

2) Assuming identical antennas (for simplicity), the mutual coupling matrix (normalized to $Z_{S}+Z_{L}$ ) is given by

$$
\mathbf{Z}=\left(\begin{array}{cc}
1 & \frac{Z_{M}}{Z_{S}+Z_{L}} \\
\frac{Z_{M}}{Z_{S}+Z_{L}} & 1
\end{array}\right)
$$

where the subscripts $S, L, M$ refer to self, load, and mutual impedances, respectively. Note that $Z_{S}$ and $Z_{M}$ are generally complex quantities [17] and that $\mathbf{Z}$ is not unitary (i.e., mutual coupling alters IXR). Suppose that the mutual coupling-free Jones matrix of the polarimeter is a real matrix, the measured vector (proportional to voltages across $Z_{L}$ on each port) is given by

$$
\mathbf{J}=\mathbf{Z}^{-1} \mathbf{J}_{L}^{\text {real }}
$$

which is a complex matrix. In this case, IXR approximation via $\mathrm{CP}$ bases may be improved by removing mutual coupling between measurements (calculating each row of $\mathbf{J}$ in the absence of the other antenna). However, as $\mathbf{Z}$ is not unitary, one should ensure that the IXR obtained does not differ significantly from the case where mutual coupling is present (as illustrated with a numerical example in the next section).

3) Nominally linear polarized antennas generally radiate fields with high but finite axial ratio. This due to the 2-or 3-dimensional current distributions on the antennas that bend around structures and are inevitably phase shifted. This effect is inherent to the antennas and cannot be compensated for in IXR approximation using CP. 


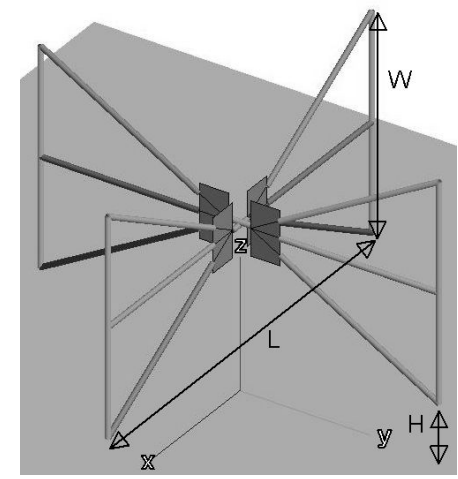

Figure 1. MWA bow-ties simulated in FEKO. Dimensions (cm): $\mathrm{L}=74$, $\mathrm{W}=40, \mathrm{H}=10$ (above an infinite perfect ground plane), feed plate $=5.5 \times 10$, feed gap $=4$, wire dia. $=0.5$. The bow-ties are aligned along $\mathrm{x}$ and $\mathrm{y}$ axes. The feed wire for the $\mathrm{y}$ dipole is $0.5 \mathrm{~cm}$ above that of the $\mathrm{x}$.

\section{Calculated Results}

This section demonstrates previous discussions with simulated results based on the MWA bow-ties [9], [10]. The antennas and their dimensions are shown in Fig. 1. The bowties were simulated in FEKO at $120 \mathrm{MHz}$ which is close to the first resonance. Each feed point was connected to the nominal LNA input impedance of $100 \Omega$. The Jones matrix was filled by exciting each feed point in turn and recording the far-field $E_{\theta}$ and $E_{\phi}$ at each direction $(\theta, \phi)$. Each source power was set to $1 \mathrm{~W}$. In every case, the LP bases were changed to $\mathrm{CP}$ via (5) to compute raw XPs.

Since the feed points are slightly offset, the antenna input impedances were slightly different $(116-j 1.89 \Omega[x]$ and $116+j 6.55 \Omega[y])$ and so were the phases of the antenna currents $\left(I_{x}=0.131 \angle 0.94^{\circ} \mathrm{A}\right.$ and $I_{y}=0.131 \angle-3.23^{\circ} \mathrm{A}$, respectively). Phase compensation is performed simply by dividing the first and second rows of $\mathbf{J}_{L}$ by $1 \angle I_{x}$ and $1 \angle I_{y}$, respectively. Tab. I shows IXR and raw XPs before and after phase compensation where significant improvement after compensation is evident. This is again clearly shown in Fig. 2 for $\theta=0-60^{\circ}$ and $\phi=0-360^{\circ}$.

\section{Table I}

CROSS-POLARIZATION ISOLATIONS AND IXR FOR THE DUAL-POLARIZED MWA BOW-TIES AT $120 \mathrm{MHZ}$ AT BORESIGHT $\left(\theta=0^{\circ}\right)$. THE FIRST AND SECOND ROWS INDICATE THE VALUES BEFORE AND AFTER PHASE COMPENSATION, RESPECTIVELY.

\begin{tabular}{c|ccc} 
& $\mathrm{XPI}_{l^{\prime}}(\mathrm{dB})$ & $\mathrm{XPI}_{r^{\prime}}(\mathrm{dB})$ & $\mathrm{IXR}(\mathrm{dB})$ \\
\hline no comp. & 28.8 & 28.8 & 55.8 \\
\hline \hline w/ comp. & 55.8 & 55.8 & 55.8 \\
\hline
\end{tabular}

Next, the effects of misaligning the $x$ bow-tie and mutual coupling are explored. The $x^{\prime}$ bow-tie was rotated by $+2^{\circ}$ about the $z$ axis with respect to $x$. The mutual coupling expressed as $\left|Z_{M} /\left(Z_{S}+Z_{L}\right)\right|$ is $-33 \mathrm{~dB}$ at this frequency. Tab. II shows IXR and XPIs at boresight with and without mutual coupling. We see that the $\mathrm{CP}$ approximation with mutual coupling, though not strictly accurate, still provides a reasonable qualitative estimate. This is again shown Fig. 3 for $\theta=0-60^{\circ}$ and $\phi=0-360^{\circ}$. When mutual coupling is removed, the $\mathrm{CP}$ approximation greatly improves, however,

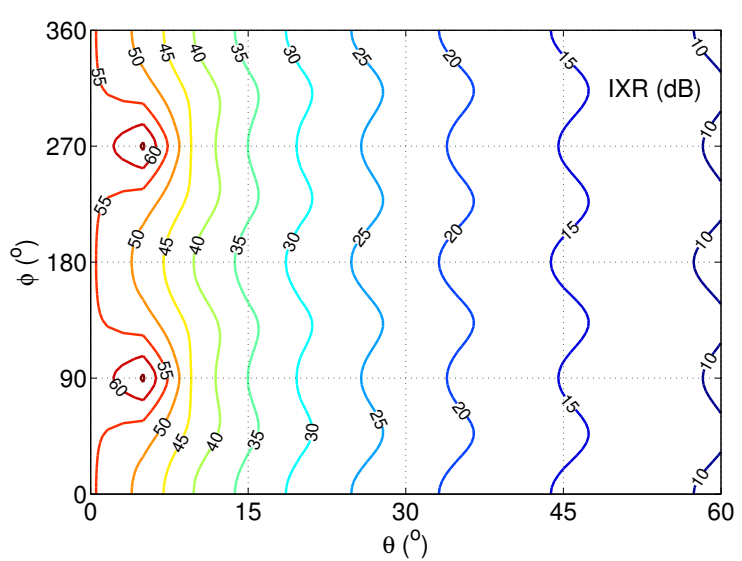

(a)

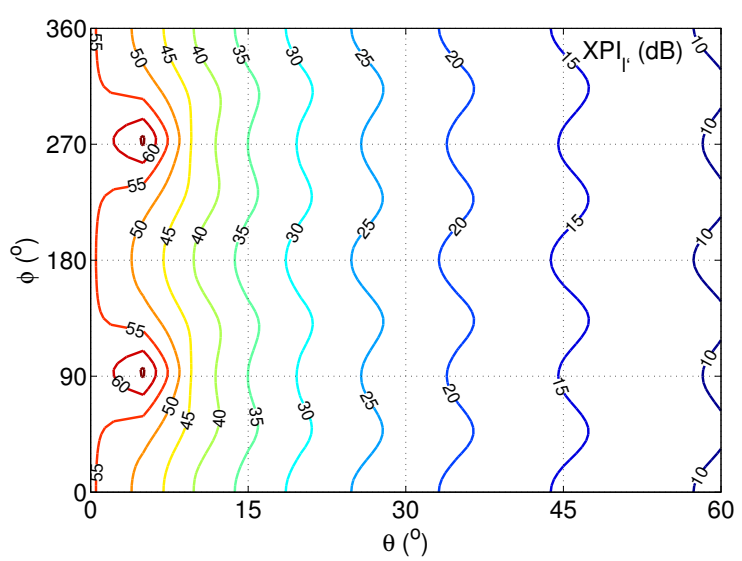

(b)

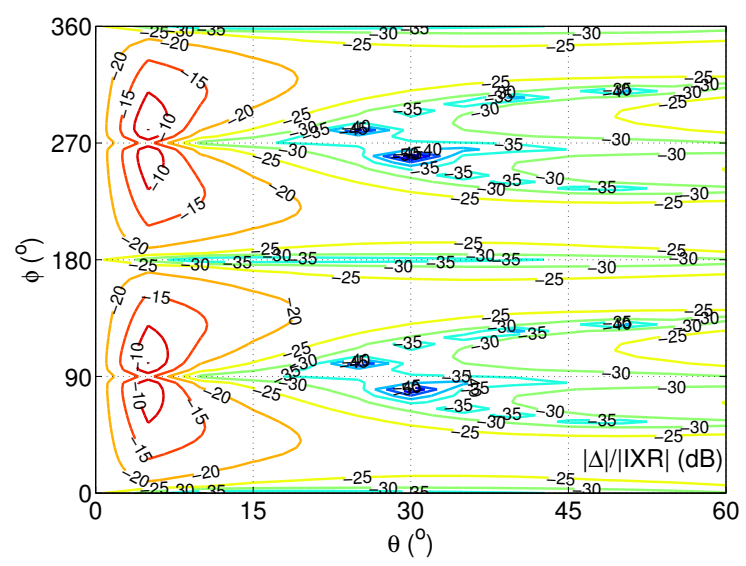

(c)

Figure 2. (a) IXR and (b) $\mathrm{XPI}_{l^{\prime}}$ contour plots for the MWA bow-ties after phase compensation. Note that the plots are virtually identical. $\mathrm{XPI}_{r^{\prime}}$ is not shown but is also virtually identical to these plots. Relative error $\mid \mathrm{XPI}_{l^{\prime}}-$ $\mathrm{IXR}|/| \mathrm{IXR} \mid$ is reported in (c).

note that IXR is slightly altered by approximately $1 \mathrm{~dB}$ at boresight (see Tab. II) from the previous case. This is again demonstrated in Fig. 4 for $\theta=0-60^{\circ}$ and $\phi=0-360^{\circ}$. These results suggest that $\mathrm{CP}$ approximation is of value in practical cases. 
Table II

CROSS-POLARIZATION ISOLATIONS AND IXR FOR THE DUAL-POLARIZED MWA BOW-TIES ( $x$ DIPOLE IS MISALIGNED BY $2^{\circ}$ ) AT $120 \mathrm{MHZ}$ AT BORESIGHT $\left(\theta=0^{\circ}\right)$ WITH PHASE COMPENSATIONS. THE FIRST ROW INDICATE VALUES OBTAINED WITH BOTH BOW-TIES PRESENT (MUTUAL COUPLING PRESENT) AND THE SECOND ROW INDICATE VALUES CALCULATED WHEN ONLY THE EXCITED BOW-TIE IS PRESENT (MUTUAL COUPLING ABSENT).

\begin{tabular}{c|ccc} 
& $\mathrm{XPI}_{l^{\prime}}(\mathrm{dB})$ & $\mathrm{XPI}_{r^{\prime}}(\mathrm{dB})$ & $\mathrm{IXR}(\mathrm{dB})$ \\
\hline w/ coupling & 32 & 31 & 36.3 \\
\hline \hline no coupling & 35.2 & 35.1 & 35.1 \\
\hline
\end{tabular}

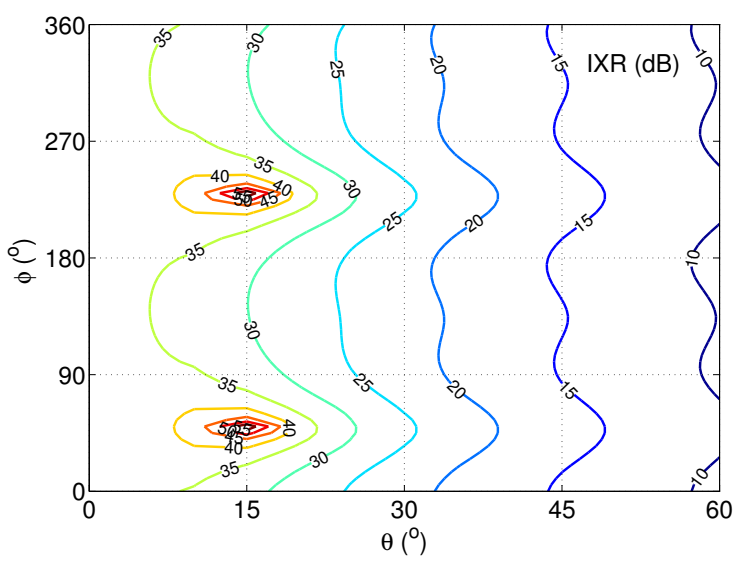

(a)

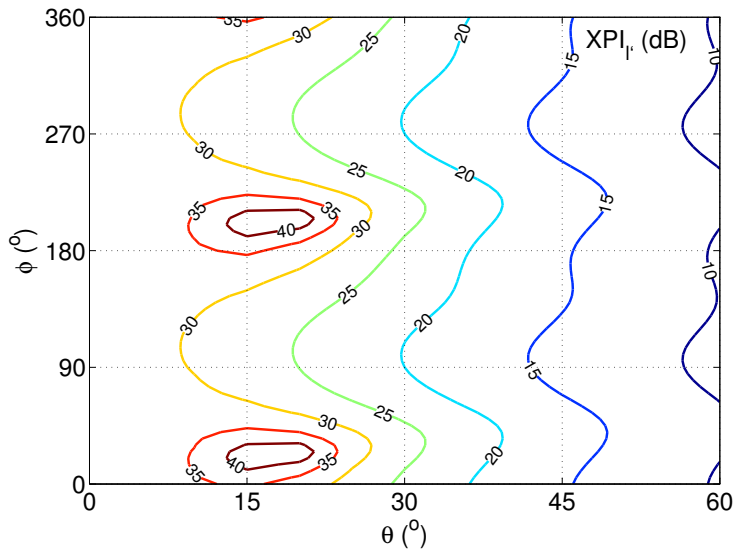

(b)

Figure 3. (a) IXR and (b) $\mathrm{XPI}_{l^{\prime}}$ contour plots for the MWA bowties ( $x$ dipole is misaligned by $2^{\circ}$, mutual coupling is present) after phase compensation. $\mathrm{XPI}_{r^{\prime}}$ is not shown but is a similar to (b).

\section{CONCLUSION}

If the Jones matrix of a dual linearly-polarized antenna is purely real and is expressed using CP bases then the IXR and raw-cross polarization ratios are identical. This condition is met for many textbook antennas, and consequently, this finding should be applicable to system calculations involving such antennas. In practical cases, where the Jones matrix of a dual-linearly-polarized antenna is not purely real, CP may still offer a good approximation if measurement phase equalization is appropriately performed. In addition, removal of mutual coupling effects may further improve the accuracy

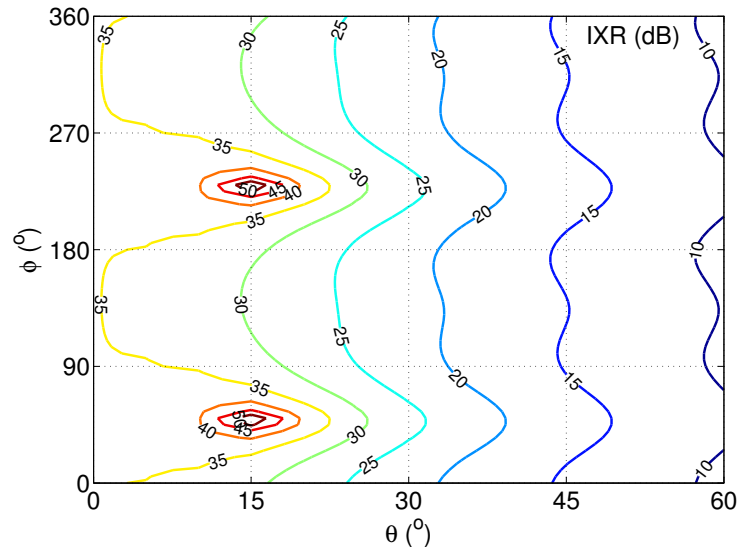

(a)

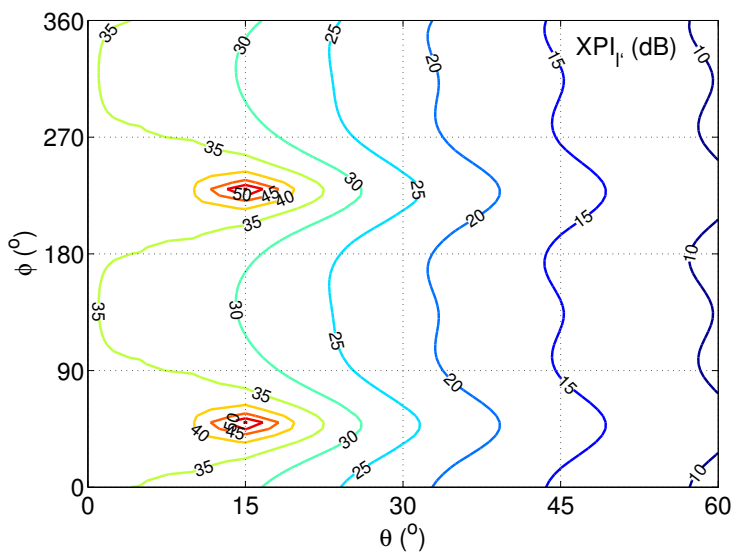

(b)

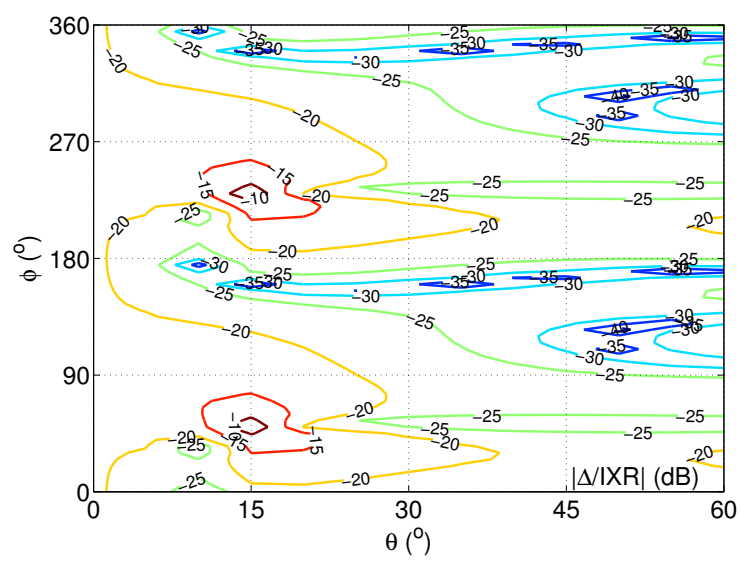

(c)

Figure 4. (a) IXR and (b) $\mathrm{XPI}_{l^{\prime}}$ contour plots for the MWA bow-ties ( $x$ dipole is misaligned by $2^{\circ}$, mutual coupling is absent) after phase compensation. Note that $\mathrm{XPI}_{l^{\prime}}$ again very closely approximates IXR. Relative error $\left|\mathrm{XPI}_{l^{\prime}}-\mathrm{IXR}\right| /|\mathrm{IXR}|$ is reported in (c).

of approximation. However, it must be done with care as the mutual coupling matrix is not unitary and does alter IXR.

\section{ACKNOWLEDGEMENT}

Discussions with A. Jiwani and J. G. bij de Vaate on this topic are gratefully acknowledged. 


\section{REFERENCES}

[1] J. P. Hamaker, J. D. Bregman, and R. J. Sault, "Understanding radio polarimetry: I. Mathematical foundations," Astronomy and Astrophysics Supplement Series, vol. 117, pp. 149-159, May 1996.

[2] R. J. Sault, J. P. Hamaker, and J. D. Bregman, "Understanding radio polarimetry: II. Instrumental calibration of an interferometer," Astronomy and Astrophysics Supplement Series, vol. 117, pp. 149-159, May 1996.

[3] S. Wijnholds, M. Ivashina, R. Maaskant, and K. Warnick, "Polarimetry with phased array antennas: Sensitivity and polarimetric performance using unpolarized sources for calibration," Antennas and Propagation, IEEE Transactions on, vol. 60, no. 10, pp. 4688 -4698, oct. 2012.

[4] T. Carozzi, "SKA polarimetry specification: Does the SKA really need to achieve ith high polarization (and sensitivity spec)?" AAVP workshop, Cambridge, 8 Dec. 2010. [Online]. Available: http://www.mrao.cam.ac.uk/projects/aavp/presentations/Carozzi_polarimetric_purity.pdf

[5] T. D. Carozzi and G. Woan, "A fundamental figure of merit for radio polarimeters," IEEE Transactions on Antennas and Propagation, vol. 59, no. 6, pp. 2058-2065, June 2011.

[6] A. J. Laub, Matrix Analysis for Scientists and Engineers. SIAM, 2005, ch. 7.

[7] M. Ivashina, O. Iupikov, R. Maaskant, W. van Cappellen, and T. Oosterloo, "An optimal beamforming strategy for wide-field surveys with phased-array-fed reflector antennas," Antennas and Propagation, IEEE Transactions on, vol. 59, no. 6, pp. $1864-1875$, june 2011.

[8] F. Keinert, "Lecture notes for Math 473," Iowa State University, Ames, Iowa, p. 7.7, August 1993.

[9] C. Lonsdale, R. Cappallo, M. Morales, F. Briggs, L. Benkevitch, J. Bowman, J. Bunton, S. Burns, B. Corey, L. deSouza, S. Doeleman, M. Derome, A. Deshpande, M. Gopala, L. Greenhill, D. Herne, J. Hewitt, P. Kamini, J. Kasper, B. Kincaid, J. Kocz, E. Kowald, E. Kratzenberg, D. Kumar, M. Lynch, S. Madhavi, M. Matejek, D. Mitchell, E. Morgan, D. Oberoi, S. Ord, J. Pathikulangara, T. Prabu, A. Rogers, A. Roshi, J. Salah, R. Sault, N. Shankar, K. Srivani, J. Stevens, S. Tingay, A. Vaccarella, M. Waterson, R. Wayth, R. Webster, A. Whitney, A. Williams, and C. Williams, "The murchison widefield array: Design overview," Proceedings of the IEEE, vol. 97, no. 8, pp. $1497-1506$, aug. 2009.

[10] S. J. Tingay, R. Goeke, J. D. Bowman, D. Emrich, S. M. Ord, D. A. Mitchell, M. F. Morales, T. Booler, B. Crosse, R. Wayth, C. J. Lonsdale, S. Tremblay, D. Pallot, T. Colegate, A. Wicenec, N. Kudryavtseva, W. Arcus, D. Barnes, G. Bernardi, F. Briggs, S. Burns, J. D. Bunton, R. J. Cappallo, B. E. Corey, A. Deshpande, L. deSouza, B. M. Gaensler, L. J. Greenhill, P. J. Hall, B. J. Hazelton, D. Herne, J. N. Hewitt, M. JohnstonHollitt, D. L. Kaplan, J. C. Kasper, B. B. Kincaid, R. Koenig, E. Kratzenberg, M. J. Lynch, S. R. McKinley, B. McWhirter, E. Morgan, D. Oberoi, J. Pathikulangara, T. Prabu, R. A. Remillard, A. E. E. Rogers, A. Roshi, J. E. Salah, R. J. Sault, N. Udaya-Shankar, F. Schlagenhaufer, K. S. Srivani, J. Stevens, R. Subrahmanyan, M. Waterson, R. L. Webster, A. R. Whitney, A. Williams, C. L. Williams, and J. S. B. Wyithe, "The Murchison Widefield Array: the Square Kilometre Array Precursor at low radio frequencies," PASA accepted: arXiv:1206.6945, June 2012.

[11] S. W. Ellingson, "Sensitivity of antenna arrays for long-wavelength radio astronomy," IEEE Transactions on Antennas and Propagation, vol. 59, no. 6, pp. 1855 - 1863, June 2011.

[12] S. J. Wijnholds and W. A. van Capellen, "In situ antenna performance evaluation of the LOFAR phased array radio telescope," IEEE Transactions on Antennas and Propagation, vol. 59, no. 6, pp. 1981 - 1989, June 2011.

[13] A. R. Parsons, D. C. Backer, G. S. Foster, M. C. H. Wright, R. F. Bradley, N. E. Gugliucci, C. R. Parashare, E. E. Benoit, J. E. Aguirre, D. C. Jacobs, C. L. Carilli, D. Herne, M. J. Lynch, J. R. Manley, and D. J. Werthimer, "The precision array for probing the epoch of re-ionization: Eight station results," The Astronomical Journal, vol. 139, no. 4, p. 1468, 2010. [Online]. Available: http://stacks.iop.org/1538-3881/139/i=4/a=1468

[14] E. de Lera Acedo, N. Razavi-Ghods, L. E. Garcia, P. Duffett-Smith, and P. Alexander, "Ultra-wideband aperture array element design for low frequency radio astronomy," IEEE Transactions on Antennas and Propagation, vol. 59, no. 6, pp. 1808 - 1816, June 2011.

[15] T. A. Milligan, Modern Antenna Design, 2nd ed. Wiley, 2005.

[16] C. Balanis, Antenna Theory: Analysis and Design, 3rd ed. Wiley, 2005.

[17] J. Richmond, "Coupled linear antennas with skew orientation," Antennas and Propagation, IEEE Transactions on, vol. 18, no. 5, pp. $694-696$, sep 1970. 